\title{
Massive Dense Nuclei in Young Starbursts: Progenitors of Early-Type Galaxy Cusps?
}

\author{
Roderik Overzier ${ }^{1}$ \\ ${ }^{1}$ Max-Planck-Institute for Astrophysics, \\ D-85748 Garching, Germany \\ Email: overzier@mpa-garching.mpg.de
}

Keywords. galaxies: starburst, galaxies: nuclei, galaxies: evolution

I present results from the Galaxy Evolution Explorer (GALEX) Key Project on "Lyman break galaxy analogs" (LBA). The LBA project was designed in order to search for nearby $(z<0.3)$ UV-luminous starburst galaxies that could serve as a training set that can be compared with starbursts at higher redshift, in particular the population of UV-selected Lyman Break Galaxies (LBGs) at $z \gtrsim 3$ (Heckman et al. 2005; Hoopes et al. 2007). This search proved highly successful and we have since shown that there exists a rare population of nearby galaxies that is most similar to LBGs in terms of stellar mass, metallicity, extinction, star formation rate, size, morphology and gas kinematics (Overzier et al. 2008, 2009ab; Basu-Zych et al. 2007, 2009).

As part of this study, we uncovered a small sample of LBA galaxies in which the UV and optical morphology is dominated by an unresolved component that is the source of a large fraction of the continuum and line emission (Overzier et al. 2009a). We have ruled out that these are unobscured (Type 1) active galactic nuclei, and have interpreted these "Dominant Central Objects" (DCOs) as being dominated by the intense light from young stars. A Starburst99 model comparison shows that the DCOs are very massive $\left(M_{*} \gtrsim 10^{9} M_{\odot}\right)$, while they are also highly compact in size $\left(R_{e} \sim 10^{2} \mathrm{pc}\right)$. The mass density of the DCOs is similar to that found in the most massive globular clusters and nuclear star clusters, but their total masses and sizes are up to two orders of magnitude larger. However, the young, dense nuclei structurally resemble the central cusps observed in typical low-mass early-type galaxies (ETGs). We suggest that some of the DCOs may be the progenitors of such ETG cusps, albeit seen at a very early stage. The host galaxies of the DCOs are further consistent with being present-day examples of the dissipative mergers at high redshift that are believed to have produced the cusps in local ETGs (e.g., Hopkins et al. 2009), as evidenced by the fact that the DCO hosts have masses comparable to those of ETGs and have disturbed morphologies indicating that the central starburst was triggered by a major inflow event. We speculate that the dense nuclei are too young at present to be growing a supermassive black hole $(\mathrm{SMBH})$ because they are still in a supernova-dominated outflow phase. However, as the DCOs appear to provide the most likely environment for growing a SMBH, i.e., deep inside the (forming) bulges of massive (forming) galaxies, we have started a campaign to search for evidence of weakly accreting black holes in the LBA and DCO samples.

\section{References}

Basu-Zych, A. R., et al. 2007, ApJS, 173, 457

Basu-Zych, A. R., et al. 2009, ApJL, 699, L118

Heckman, T. M., et al. 2005, ApJL, 619, L35

Hoopes, C., et al. 2007, ApJS, 173, 441

Hopkins, P., et al. 2009, ApJS, 181, 135

Overzier, R. A., et al. 2008, ApJ, 677, 37

Overzier, R. A., et al. 2009a, ApJ, 706, 203

Overzier, R. A., et al. 2009b, submitted to ApJ (arXiv:0911.1279) 